\title{
Diagnostic performance of the combined nasal and throat swab in patients admitted to hospital with suspected COVID-19
}

Kuan Ken Lee ${ }^{1 *} \mathbb{D}$, Dimitrios Doudesis ${ }^{1,2}$, Daniella A. Ross ${ }^{3}$, Anda Bularga ${ }^{1}$, Claire L. MacKintosh ${ }^{3}$, Oliver Koch $^{3}$, Ingolfur Johannessen ${ }^{4}$, Kate Templeton ${ }^{4}$, Sara Jenks ${ }^{5}$, Andrew R. Chapman ${ }^{1}$, Anoop S. V. Shah ${ }^{1,6,7}$, Atul Anand ${ }^{1}$, Meghan R. Perry ${ }^{3}$, Nicholas L. Mills ${ }^{1,2}$ and on behalf of the DataLoch COVID-19 Collaboration

\begin{abstract}
Background: Accurate diagnosis in patients with suspected coronavirus disease 2019 (COVID-19) is essential to guide treatment and limit spread of the virus. The combined nasal and throat swab is used widely, but its diagnostic performance is uncertain.

Methods: In a prospective, multi-centre, cohort study conducted in secondary and tertiary care hospitals in Scotland, we evaluated the combined nasal and throat swab with reverse transcriptase-polymerase chain reaction (RT-PCR) for severe acute respiratory syndrome coronavirus-2 (SARS-CoV-2) in consecutive patients admitted to hospital with suspected COVID-19. Diagnostic performance of the index and serial tests was evaluated for a primary outcome of confirmed or probable COVID-19, and a secondary outcome of confirmed COVID-19 on serial testing. The diagnosis was adjudicated by a panel, who recorded clinical, laboratory and radiological features blinded to the test results.

Results: We enrolled 1368 consecutive patients (median age 68 [interquartile range, IQR 53-80] years, 47\% women) who underwent a total of 3822 tests (median 2 [IQR 1-3] tests per patient). The primary outcome occurred in 36\% (496/1368), of whom 65\% (323/496) and 35\% (173/496) had confirmed and probable COVID-19, respectively. The index test was positive in 255/496 (51\%) patients with the primary outcome, giving a sensitivity and specificity of $51.4 \%$ (95\% confidence interval [Cl] 48.8 to $54.1 \%$ ) and $99.5 \%$ (95\% Cl 99.0 to $99.8 \%$ ). Sensitivity increased in those undergoing 2, 3 or 4 tests to $60.1 \%$ (95\% Cl 56.7 to $63.4 \%), 68.3 \%$ (95\% Cl 64.0 to $72.3 \%)$ and $77.6 \%$ (95\% Cl 72.7 to $81.9 \%$ ), respectively. The sensitivity of the index test was $78.9 \%$ (95\% Cl 74.4 to $83.2 \%$ ) for the secondary outcome of confirmed COVID-19 on serial testing.
\end{abstract}

Conclusions: In patients admitted to hospital, a single combined nasal and throat swab with RT-PCR for SARS-CoV2 has excellent specificity, but limited diagnostic sensitivity for COVID-19. Diagnostic performance is significantly improved by repeated testing.

Keywords: Coronavirus disease 2019, Reverse transcriptase-polymerase chain reaction test, Diagnostic evaluation

\footnotetext{
* Correspondence: ken.lee@ed.ac.uk

${ }^{1}$ BHF Centre for Cardiovascular Science, University of Edinburgh, Edinburgh EH16 4SA, UK

Full list of author information is available at the end of the article
}

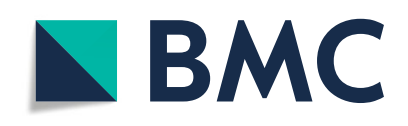

(- The Author(s). 2021 Open Access This article is licensed under a Creative Commons Attribution 4.0 International License, which permits use, sharing, adaptation, distribution and reproduction in any medium or format, as long as you give appropriate credit to the original author(s) and the source, provide a link to the Creative Commons licence, and indicate if changes were made. The images or other third party material in this article are included in the article's Creative Commons licence, unless indicated otherwise in a credit line to the material. If material is not included in the article's Creative Commons licence and your intended use is not permitted by statutory regulation or exceeds the permitted use, you will need to obtain permission directly from the copyright holder. To view a copy of this licence, visit http://creativecommons.org/licenses/by/4.0/. The Creative Commons Public Domain Dedication waiver (http://creativecommons.org/publicdomain/zero/1.0/) applies to the data made available in this article, unless otherwise stated in a credit line to the data. 


\section{Background}

Severe acute respiratory syndrome coronavirus-2 (SARS$\mathrm{CoV}-2$ ) is a novel coronavirus, which is responsible for the global pandemic of coronavirus disease 2019 (COVID-19) [1, 2]. Timely and accurate diagnostic testing in patients with suspected COVID-19 is essential to guide treatment and implement infection control measures to limit spread of the virus.

Reverse transcriptase-polymerase chain reaction (RTPCR) assays on material collected by swabbing the nasopharynx and oropharynx, herein referred to as the combined nose and throat swab, are the most commonly used diagnostic tests [3]. However, a number of reports have indicated discordance between the results of testing and clinical or radiological findings in patients with symptoms of suspected COVID-19 and increasingly clusters of asymptomatic carriers of SARS-CoV-2 are recognised [4-8]. As such, uncertainty remains as to the diagnostic performance of the combined nasal and throat swab to diagnose the clinical condition of COVID-19, particularly in patients presenting late following the onset of symptoms when the viral load may be lower.

Our aim was to evaluate the performance of the combined nasal and throat swab for the clinical diagnosis of COVID-19 in consecutive patients admitted to hospital with suggestive symptoms, and to determine whether there is heterogeneity across subgroups.

\section{Methods}

\section{Study design}

In a prospective, multi-centre, cohort study in secondary and tertiary care hospitals in Scotland, United Kingdom, we evaluated the diagnostic performance of the combined nasal and throat swab with RT-PCR for SARSCoV-2 in consecutive patients admitted to hospital for symptoms of suspected COVID-19. The study was performed with approval of the local Research Ethics Committee and delegated Caldicott Guardian for the National Health Service (NHS) Lothian Health Board, in accordance with the Declaration of Helsinki. All data were collected from the patient record and national registries, deidentified and linked in a data repository (DataLoch, Edinburgh, United Kingdom) within a secure safe haven. To ensure that every eligible patient was included and avoid selection bias, consent was not sought from individual patients. Only summary data was extracted to minimise the risk of disclosure.

\section{Participants}

Consecutive adult patients $\geq 18$ years old were identified by the attending clinician using an electronic form integrated into the care pathway at the time of testing with the combined nasal and throat swab. Patients were eligible for inclusion if they were admitted to hospital with symptoms suggestive of COVID-19 and had a reportable SARS-CoV-2 RT-PCR result from material obtained through the combined swab (Fig. 1). Patients were excluded if they had no symptoms and testing was performed for screening purposes only, or if they had a previous diagnosis of COVID-19.

\section{Procedures}

During the study period RT-PCR for SARS-CoV-2 was performed on material obtained from a combined nasal and throat swab in patients presenting with symptoms suggestive of COVID-19 who were admitted to hospital. Repeat testing was at the discretion of the clinician responsible for care. Although patients were enrolled and underwent assessment and sampling at three hospitals in the region, RT-PCR was performed in a single regional virology laboratory at the Royal Infirmary of Edinburgh. All specimens were extracted using a single standardised protocol. Material obtained from the combined nose and throat swabs were added to a viral transport media (Remel MicroTest M4RT). Subsequently, an automated NucliSENS $^{\odot}$ easyMag (bioMérieux) platform was used to obtain $110 \mu \mathrm{L}$ of eluate containing purified ribonucleic acid (RNA) after $200 \mu \mathrm{L}$ of the sample was added to $1 \mathrm{~mL}$ of easyMAG lysis buffer. The vast majority of tests (94\%) were performed using a modified in-house RT-PCR [9] and only a small number of samples (6\%) were analysed using the AllplexTM2019- nCoV Assay from SeeGene (Seoul, South Korea) [10].

An electronic form was embedded into the order of the combined nasal and throat swab to prospectively record information on the indication for testing, symptom type, and duration of symptoms. This form was completed by the usual care clinician at the time of testing. Data was extracted from the electronic patient record (TrakCare; InterSystems Corporation, Cambridge, MA, USA), laboratory information management system (iLaboratory, Advanced Expert Systems Medical, Derby, United Kingdom), the Scottish Morbidity Record, the Scottish Drug Dispensing Database, and the Scottish Care Information store. The Scottish Index of Multiple Deprivation (SIMD), an area-based measure of deprivation, was used to define socioeconomic status of each individual based on 31 indicators across 7 domains (income, employment, health, education, skills and training, housing, geographic access, and crime) [11].

\section{Outcomes}

Diagnostic performance of the index test was evaluated for a primary outcome of probable or confirmed COVID-19, and a secondary outcome of confirmed COVID-19 on serial testing. All clinical diagnoses were adjudicated by an independent, inter-disciplinary panel 


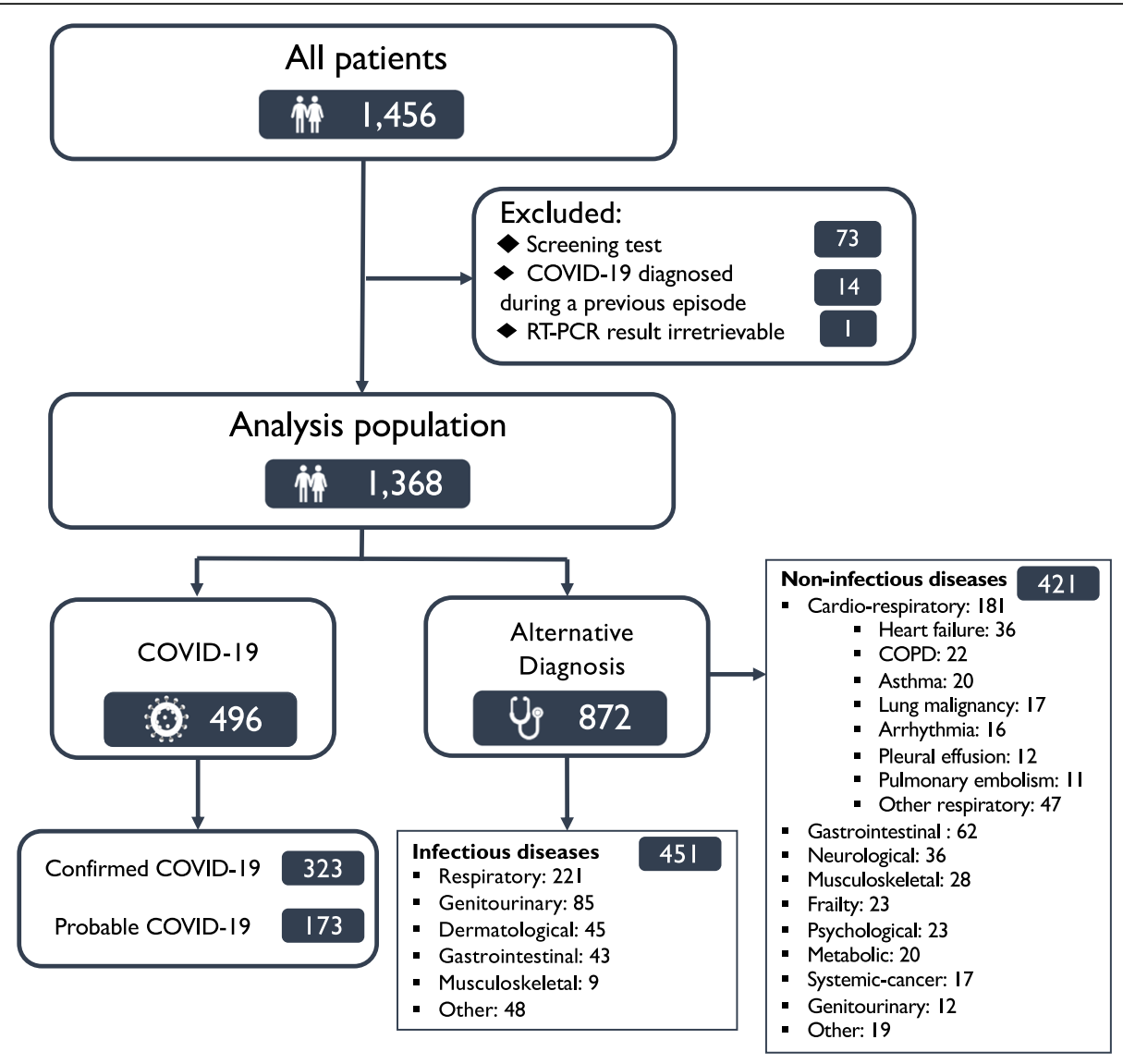

Fig. 1 Flow diagram of study population

of clinicians using all information available within the electronic patient record, including contact history and review of all laboratory and radiological imaging performed.

The diagnosis of COVID-19 was based on the case definition proposed by the World Health Organisation [12]. The panel identified patients as suspected COVID19 where they were admitted to hospital with an acute respiratory illness (fever with at least one sign or symptom of respiratory disease such as cough or shortness of breath) and had no alternative diagnosis that fully explained the clinical presentation. The panel recorded clinical, laboratory and radiological features of suspected COVID-19 without knowledge of the index and subsequent test results. Patients with parameters consistent with COVID-19 were subsequently classified as probable COVID-19 where all tests for the SARS-CoV-2 virus from any sample type were negative, or confirmed COVID-19 where any test was positive during the hospital episode, or within 7 days of the index presentation in those discharged.

For the evaluation of diagnostic performance for the primary outcome, patients were classified into the following groups: 1) Confirmed COVID-19 in those with acute respiratory illness AND a positive test for SARSCoV-2 (true positives); 2) Probable COVID-19 in those with acute respiratory illness AND negative tests for SARS-CoV-2 AND no other diagnosis to explain the clinical presentation (false negatives); 3) Alternative diagnosis that fully explained their clinical presentation AND a positive test for SARS-CoV-2 (false positive); or 4) Alternative diagnosis that fully explained their clinical presentation AND negative tests for SARS-CoV-2 (true negatives).

For evaluation of diagnostic performance for the secondary outcome, patients with confirmed COVID-19 were classified as true positives, and those with probable COVID-19 were classified as true negatives rather than false negatives.

\section{Sample size and power}

Based on data from the Hubei and Shandong provinces and Beijing, China, we anticipated that approximately $32 \%(126 / 398)$ of combined nasal and throat swabs performed would be positive for SARS-CoV-2 [6]. We recognise that there may be differences in the approach to the selection of patients for testing between countries, and therefore our sample size was based on a more 
conservative positive test rate of $20 \%$. We estimated that with 1000 patients, we would have $80 \%$ power to estimate the confidence interval for a sensitivity of $90 \%$ with lower and upper intervals of 85 and 95\% respectively.

\section{Statistical analysis}

Baseline characteristics, clinical features, and laboratory results are summarised as number (percentage) or median (interquartile range) for the study population, and stratified according to the adjudicated diagnosis. Quantitative variables were compared in patients with and without the primary and secondary outcomes using parametric (Student $t$ test) and non-parametric (MannWhitney $\mathrm{U}$ ) tests as appropriate, and qualitative variables were compared using either Chi-squared test or Fisher's exact test as appropriate. Statistical significance was set at $P<0.05$. Two-by-two contingency tables were constructed to compare the index test (positive or negative) in those with and without the primary and secondary outcome on serial testing (reference standard). Test sensitivity, specificity, negative predictive value (NPV) and positive predictive value (PPV) with 95\% confidence intervals was determined in all participants and in prespecified subgroups including age, sex, duration of symptoms prior to testing, fever, and respiratory tract symptoms. In patients where more than one test was performed, we report test results and compare diagnostic performance of the index test with the performance of multiple tests. All analyses were performed using $\mathrm{R}$ (version 3.6.1).

\section{Results}

Between April 3 and 20, 2020, we enrolled 1368 consecutive patients (median age 68 [interquartile range, IQR 53-80] years, $47 \%$ women) who underwent a total of 3822 combined nasal and throat swab tests (median 2 [IQR 1-3] tests per patient) for symptoms of suspected COVID-19 (Fig. 1). The primary outcome occurred in $36 \%(496 / 1368)$, of whom 65\% (323/496) and 35\% (173/ 496) had confirmed and probable COVID-19, respectively. Of those with an alternative diagnosis (64\% [872/ 1269]), the most frequent diagnoses were other respiratory infections $(25 \%,[221 / 872])$ and non-communicable cardiorespiratory conditions (21\%, [181/872]), such as heart failure, chronic obstructive pulmonary disease and asthma.

Patients with confirmed or probable COVID-19 were older than those with an alternative diagnosis (median age of 71 [57-82] versus 67 [53-80] years), and were less likely to be from an area of deprivation (13\% [65/496] versus $20 \%$ [174/872]). However, they had similar comorbidities and were as likely to be receiving angiotensin converting enzyme inhibitors, corticosteroids or immunosuppressants at presentation (Table 1).
Compared to patients with an alternative diagnosis, those with confirmed or probable COVID-19 had a lower lymphocyte and neutrophil count (median $1.14 \times$ $10^{9} / \mathrm{L}$ versus $1.44 \times 10^{9} / \mathrm{L}$ and $5.8 \times 10^{9} / \mathrm{L}$ versus $6.4 \times$ $10^{9} / \mathrm{L}$, respectively), but a higher C-reactive protein concentration $(52 \mathrm{mg} / \mathrm{dL}$ versus $22 \mathrm{mg} / \mathrm{dL})$ at presentation (eTable 1 in the Supplement). In patients with probable or confirmed COVID-19, compared to those with an alternative diagnosis, some symptoms and signs were more common, including fever on presentation $(65 \%$ [322/496] versus $42 \%$ [369/872]), upper and lower respiratory tract symptoms $(13 \%$ [63/496] versus 9\% [78/ 872]; 85\% [419/496] versus 54\% [473/872], respectively), and systemic symptoms (52\% [257/496] versus $28 \%$ [244/872]). In contrast, there were no differences in the frequency of neurological (24\% [118/496] versus 22\% [191/872]) or gastrointestinal symptoms (22\% [107/496] versus 24\% [209/872]) (Table 1 and Fig. 2a). Patients with probable or confirmed COVID-19 were six-times more likely to have radiological signs of infection on chest imaging than those with an alternative diagnosis (64\% [316/496] versus 11\% [93/872]). Patients with confirmed COVID-19 had similar symptoms as those with probable COVID-19 (Fig. 2b and eTable 2 in the Supplement), but were more likely to have lymphopenia, systemic inflammation and radiological signs of infection (eTable 3 in the Supplement).

The index test was positive in $255 / 496$ (51\%) patients with the primary outcome (eTable 2 in the Supplement), giving a sensitivity, specificity, negative predictive value and positive predictive value of $51.4 \%$ (95\% confidence interval [CI] 48.8 to $54.1 \%$ ), $99.5 \%$ (95\% CI 99.0 to 99.8\%), $78.3 \%$ (95\% CI 76.0 to $80.4 \%$ ) and $98.5 \%$ (95\% CI 97.7 to $99.0 \%$ ), respectively (Table 2 ). Sensitivity was lower in patients from areas with the greatest deprivation (32.6\% [95\% CI 21.6 to $43.9 \%$ ] versus $53.9 \%$ [95\% CI 45.7 to $62.1 \%$ ], quintile with the most deprivation compared to the quintile with the least deprivation). Otherwise, sensitivity remained consistent across other patient demographic factors, comorbidities and symptoms (Fig. 3a). The negative predictive value of the index test was consistent across patient demographics and comorbidities, but was lower in those with fever (70.3\% [95\% CI 66.3 to 74.2\%]), lower respiratory symptoms $(69.5 \%$ [95\% CI 66.1 to $73.0 \%])$ and those with a longer duration of symptoms prior to hospital admission (68.7\% [95\% CI 63.2 to $74.1 \%$ ] versus $87.1 \%$ [95\% CI 83.3 to $90.7 \%$ ] for patients presenting $\geq 4$ days after symptom onset compared to those presenting within 1 day) (Fig. 3b).

The majority of patients underwent serial testing (59.6\%, 815/1368) with $22.7 \%$ (310/1368) undergoing 4 or more serial tests (eFigure 1 and 2, and eTable 4 in the Supplement). Of those with confirmed COVID-19, 
Table 1 Baseline characteristics of patients undergoing testing with suspected COVID-19

\begin{tabular}{|c|c|c|c|c|}
\hline & $\begin{array}{l}\text { All } \\
(n=1368)\end{array}$ & $\begin{array}{l}\text { COVID-19a } \\
(n=496) \\
\end{array}$ & $\begin{array}{l}\text { Alternative diagnosis } \\
(n=872)\end{array}$ & $P$-value \\
\hline Age, years & $68(53,80)$ & $71(57,82)$ & $67(51,78)$ & $<0.001$ \\
\hline Sex & & & & 0.078 \\
\hline Men & 731 (53\%) & $281(57 \%)$ & $450(52 \%)$ & \\
\hline Women & $637(47 \%)$ & $215(43 \%)$ & $422(48 \%)$ & \\
\hline Ethnicity & & & & 0.83 \\
\hline White & 1087 (97\%) & $372(97 \%)$ & 715 (98\%) & \\
\hline Other & $29(2.6 \%)$ & $11(2.9 \%)$ & $18(2.5 \%)$ & \\
\hline Deprivation $^{\mathrm{b}}$ & & & & 0.002 \\
\hline 1 (most deprivation) & $239(18 \%)$ & $65(13 \%)$ & $174(20 \%)$ & \\
\hline 2 & $321(24 \%)$ & $116(24 \%)$ & $205(24 \%)$ & \\
\hline 3 & $236(17 \%)$ & $79(16 \%)$ & $157(18 \%)$ & \\
\hline 4 & $243(18 \%)$ & $93(19 \%)$ & $150(17 \%)$ & \\
\hline 5 (least deprivation) & $319(23 \%)$ & $139(28 \%)$ & $180(21 \%)$ & \\
\hline Duration of symptoms, days & $3.0[1.0-5.0]$ & $3.0[2.0-7.0]$ & $2.0[1.0-4.0]$ & $<0.001$ \\
\hline \multicolumn{5}{|l|}{ Clinical features } \\
\hline Fever & $691(51 \%)$ & $322(65 \%)$ & 369 (42\%) & $<0.001$ \\
\hline Upper respiratory tract symptoms & $141(10 \%)$ & $63(13 \%)$ & $78(9 \%)$ & 0.034 \\
\hline Lower respiratory tract symptoms & 891 (65\%) & 419 (85\%) & $472(54 \%)$ & $<0.001$ \\
\hline Systemic symptoms & $501(37 \%)$ & $257(52 \%)$ & $244(28 \%)$ & $<0.001$ \\
\hline Neurological symptoms & $309(23 \%)$ & $118(24 \%)$ & $191(22 \%)$ & 0.45 \\
\hline Gastrointestinal symptoms & $316(23 \%)$ & $107(22 \%)$ & 209 (24\%) & 0.36 \\
\hline Lymphopenia & $894(65 \%)$ & $406(82 \%)$ & $488(56 \%)$ & $<0.001$ \\
\hline Inflammation & $986(72 \%)$ & $421(86 \%)$ & $565(65 \%)$ & $<0.001$ \\
\hline Radiological signs & $409(30 \%)$ & $316(64 \%)$ & $93(11 \%)$ & $<0.001$ \\
\hline \multicolumn{5}{|l|}{ Comorbidities } \\
\hline Diabetes mellitus & $251(18 \%)$ & $96(19 \%)$ & $155(18 \%)$ & 0.51 \\
\hline Ischemic heart disease & $136(10 \%)$ & $45(9.1 \%)$ & $91(11 \%)$ & 0.44 \\
\hline Heart failure & $112(8.3 \%)$ & $38(7.7 \%)$ & $74(8.6 \%)$ & 0.62 \\
\hline Stroke & $82(6.0 \%)$ & $27(5.4 \%)$ & $55(6.3 \%)$ & 0.60 \\
\hline COPD & $206(15 \%)$ & $58(12 \%)$ & $148(17 \%)$ & 0.011 \\
\hline Asthma & $113(8.3 \%)$ & $30(6.0 \%)$ & $83(9.5 \%)$ & 0.033 \\
\hline Liver cirrhosis & $27(2.0 \%)$ & $8(1.6 \%)$ & $19(2.2 \%)$ & 0.60 \\
\hline End stage kidney disease & $25(1.8 \%)$ & $12(2.4 \%)$ & $13(1.5 \%)$ & 0.31 \\
\hline \multicolumn{5}{|l|}{ Medications at presentation } \\
\hline ACE inhibitors or ARBs & $336(25 \%)$ & $129(26 \%)$ & 207 (24\%) & 0.38 \\
\hline Corticosteroids & $223(16 \%)$ & $73(15 \%)$ & $150(17 \%)$ & 0.27 \\
\hline Immunosuppressants & $34(2.5 \%)$ & $15(3.0 \%)$ & 19 (2.2\%) & 0.43 \\
\hline
\end{tabular}

Values are No. (\%) or median [inter-quartile range]

Abbreviations: $A C E$ angiotensin converting enzyme, $A R B$ angiotensin receptor blockers, COPD chronic obstructive pulmonary disease

${ }^{a}$ Confirmed or probable COVID-19 with no alternative diagnosis

${ }^{b}$ Scottish Index of Multiple Deprivation (SIMD) quintiles

$\neq$ Comparison between patients with COVID-19 versus those with alternative diagnosis

patients with a negative index test result underwent more serial testing than those with a positive index test result $(95.6 \%$ [65/68] versus 65.9\% [168/255] with median test per patient of 4 [2-6] versus 2 [1-5] respectively). The median time between first and second tests was shorter in patients with confirmed COVID-19 


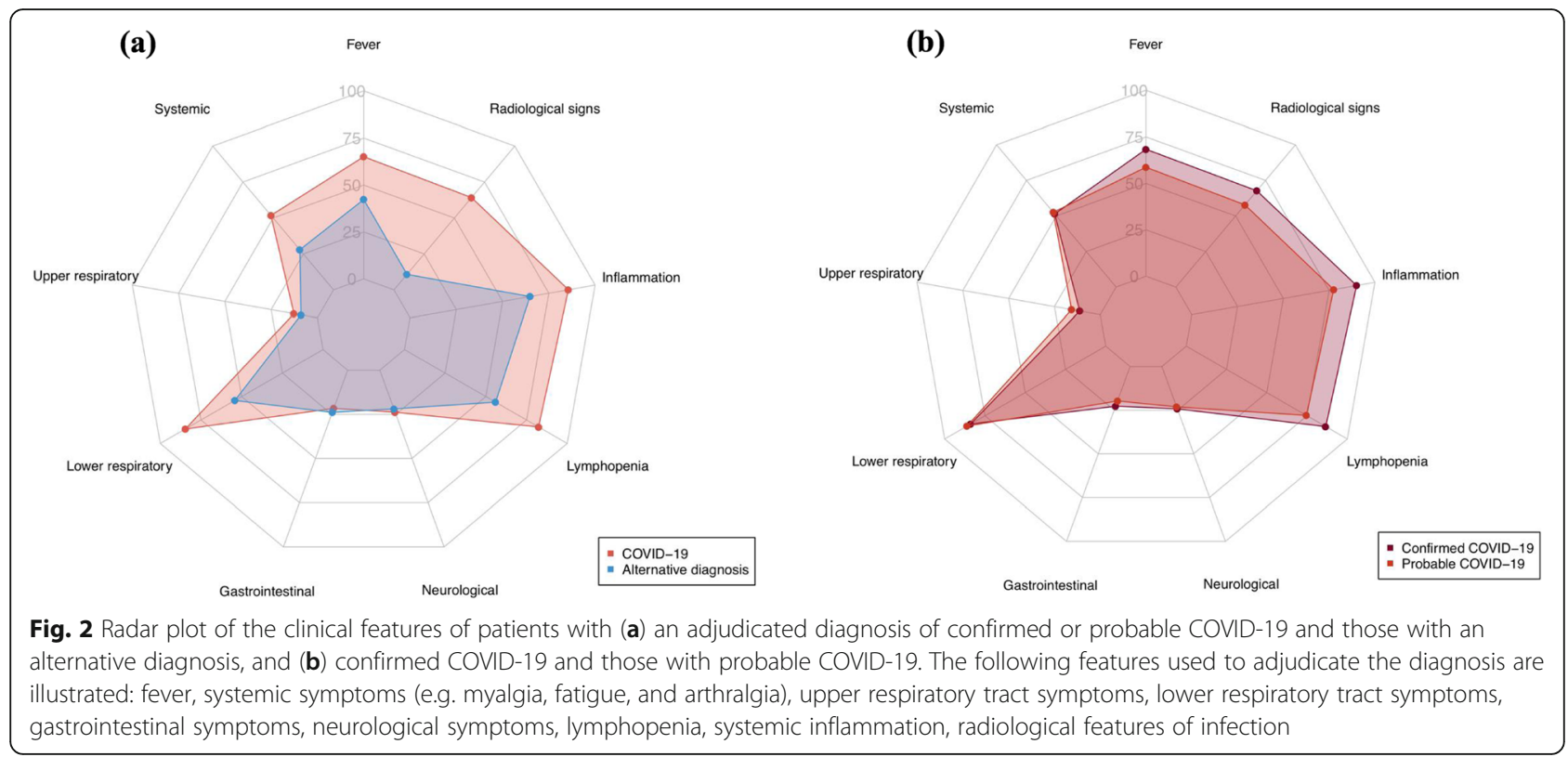

who had a negative index test result and those with probable COVID-19 (1.7 [0.8-10.9] days and 1.2 [0.94.6] days respectively) compared to patients with confirmed COVID-19 who had a positive index test result and those with an alternative diagnosis (6.8 [4.0-8.6] days and 6.1 [1.1-21.8] days respectively). Sensitivity for the primary outcome increased in those undergoing 2,3 or 4 serial tests to $60.1 \%$ (95\% CI 56.7 to $63.4 \%$ ), $68.3 \%$ (95\% CI 64.0 to $72.3 \%$ ) and $77.6 \%$ (95\% CI 72.7 to $81.9 \%$ ), respectively (Table 2 and Fig. 4). The negative predictive value increased more modestly on serial testing; from $78.3 \%$ (76.0 to $80.4 \%$ ) for the index test to $79.8 \%$ (75.0 to $83.9 \%$ ) in those undergoing 4 serial tests. These observations persisted in a sensitivity analysis restricted to patients who underwent at least 4 tests and for the secondary outcome (Fig. 4, eFigure 3 and 4, and eTable 5 in the Supplement).

Sensitivity of the index test for the secondary outcome of a diagnosis of confirmed COVID-19 on serial testing was $78.9 \%$ (95\% CI 74.4 to $83.2 \%$ ) (eFigure $5 \mathrm{a}$ in the Supplement). There was no significant heterogeneity in sensitivity across patient demographics, comorbidities or

Table 2 Diagnostic performance of the index and serial combined nasal and throat swab for the primary outcome of a diagnosis of confirmed or probable COVID-19

\begin{tabular}{|c|c|c|c|c|c|c|c|c|}
\hline $\begin{array}{l}\text { Serial } \\
\text { tests }\end{array}$ & $\begin{array}{l}\text { True } \\
\text { negative }\end{array}$ & $\begin{array}{l}\text { False } \\
\text { negative }\end{array}$ & $\begin{array}{l}\text { True } \\
\text { positive }\end{array}$ & $\begin{array}{l}\text { False } \\
\text { positive }\end{array}$ & $\begin{array}{l}\text { Sensitivity } \\
(95 \% \mathrm{Cl})\end{array}$ & $\begin{array}{l}\text { Negative predictive } \\
\text { value } \\
(95 \% \mathrm{Cl})\end{array}$ & $\begin{array}{l}\text { Positive predictive } \\
\text { value } \\
(95 \% \mathrm{Cl})\end{array}$ & $\begin{array}{l}\text { Specificity } \\
(95 \% \mathrm{Cl})\end{array}$ \\
\hline $\begin{array}{l}1 \text { (index } \\
\text { test) }\end{array}$ & 868 & 241 & 255 & 4 & $\begin{array}{l}51.4(48.8- \\
54.1)\end{array}$ & $78.3(76.0-80.4)$ & 98.5 (97.7-99) & $\begin{array}{l}99.5(99.0- \\
99.8)\end{array}$ \\
\hline 2 & 459 & 141 & 212 & 5 & $\begin{array}{l}60.1(56.7- \\
63.4)\end{array}$ & $76.5(73.5-79.3)$ & 97.7 (96.4-98.5) & $\begin{array}{l}98.9 \text { (98.0- } \\
99.4)\end{array}$ \\
\hline 3 & 235 & 76 & 164 & 7 & $\begin{array}{l}68.3(64.0- \\
72.3)\end{array}$ & $75.6(71.5-79.2)$ & 95.9 (93.7-97.3) & $\begin{array}{l}97.1(95.2- \\
98.3)\end{array}$ \\
\hline 4 & 142 & 36 & 125 & 7 & $\begin{array}{l}77.6(72.7- \\
81.9)\end{array}$ & $79.8(75.0-83.9)$ & 94.7 (91.6-96.7) & $\begin{array}{l}95.3(92.3- \\
97.2)\end{array}$ \\
\hline 5 & 94 & 24 & 94 & 6 & $\begin{array}{l}79.7(73.8- \\
84.5)\end{array}$ & 79.7 (73.8-84.5) & $94.0(90.0-96.5)$ & $\begin{array}{l}94.0(90.0- \\
96.5)\end{array}$ \\
\hline 6 & 62 & 11 & 75 & 6 & $\begin{array}{l}87.2(81.0- \\
91.6)\end{array}$ & $84.9(78.4-89.7)$ & $92.6(87.3-95.8)$ & $\begin{array}{l}91.2(85.6- \\
94.7)\end{array}$ \\
\hline 7 & 46 & 10 & 55 & 6 & $\begin{array}{l}84.6(77.0- \\
90.0)\end{array}$ & $82.1(74.2-88.0)$ & $90.2(83.4-94.3)$ & $\begin{array}{l}88.5(81.4- \\
93.1)\end{array}$ \\
\hline 8 & 31 & 6 & 43 & 4 & $\begin{array}{l}87.8(79.1- \\
93.2)\end{array}$ & $83.8(74.5-90.2)$ & 91.5 (83.6-95.8) & $\begin{array}{l}88.6(80.0- \\
93.7)\end{array}$ \\
\hline
\end{tabular}




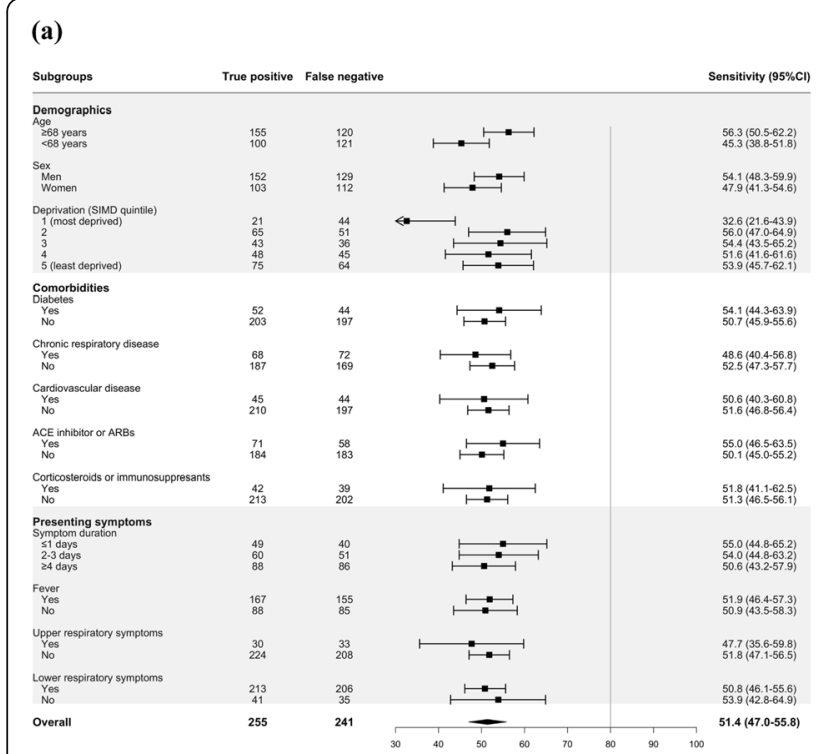

(b)

Fig. 3 Forest plot of the (a) sensitivity and (b) negative predictive value of the index combined nasal and throat swab for a diagnosis of confirmed or probable COVID-19 stratified by subgroups

presenting symptoms. The negative predictive value of the index test was $93.8 \%$ (95\% CI 92.4 to $95.2 \%$ ) for a diagnosis of confirmed COVID-19 on serial testing (eFigure $5 \mathrm{~b}$ in the Supplement). The negative predictive value remained consistent across patient demographics and comorbidities, but was lower in patients who presented with a fever $(89.6 \%$ [95\% CI 86.9 to $92.1 \%$ ]) and those who had lower respiratory symptoms $(91.5 \%$ [95\% CI 89.4 to $93.6 \%])$.

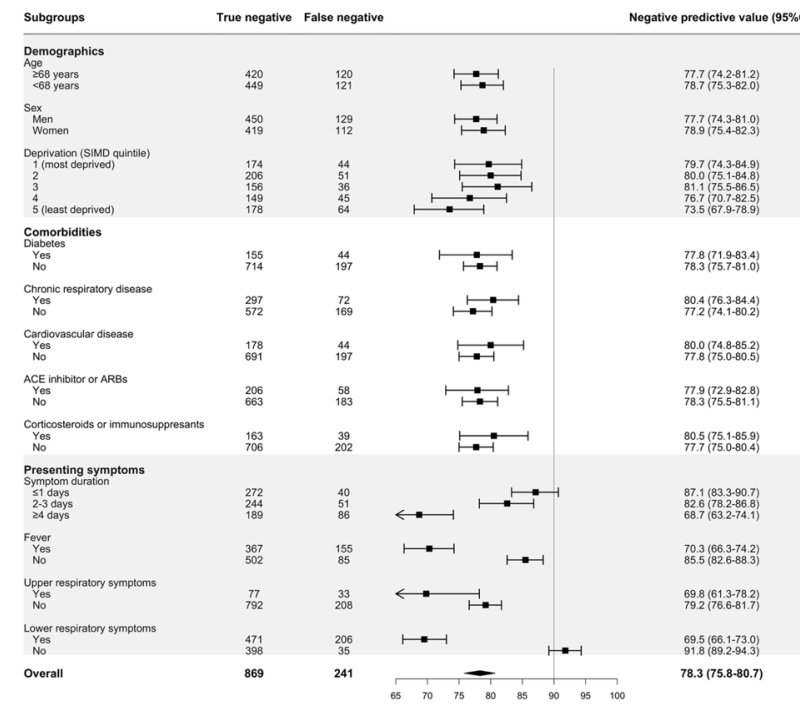

\section{Discussion}

In this prospective, multi-centre, cohort study, we evaluated the diagnostic performance of the combined nasal and throat swab with RT-PCR for SARS-CoV-2 in consecutive patients admitted to hospital with symptoms of suspected COVID-19. We report a number of potentially important findings. First, a single test had excellent specificity, but limited sensitivity for an adjudicated diagnosis of probable or confirmed COVID-19. Second, the

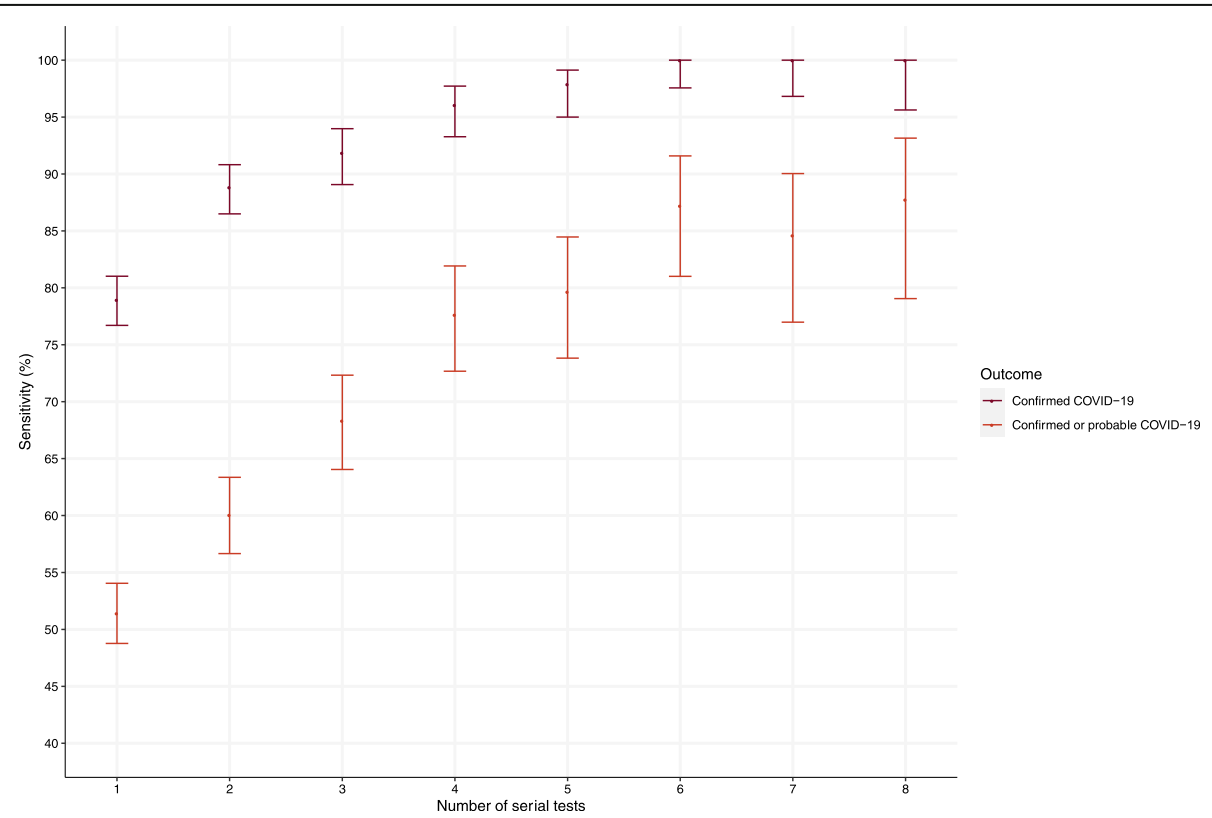

Fig. 4 Sensitivity of serial testing using the combined nasal and throat swab for the primary (confirmed or probable COVID-19) and secondary (confirmed COVID-19) outcome 
sensitivity of the index test was higher for patients with confirmed COVID-19 on serial testing, but still missed 1 in 5 patients with the diagnosis. Third, diagnostic performance was similar in most patient subgroups, but the sensitivity was lower in those from more deprived areas, and the negative predictive value was lower in those presenting later following the onset of symptoms, and in those with fever or lower respiratory symptoms. Finally, we observed a significant improvement in diagnostic sensitivity with repeated testing on up to four occasions.

Our study has several strengths. This was a prospective, multi-centre study that was adequately powered to evaluate the diagnostic performance of the combined nasal and throat swab. Patients were enrolled using an electronic form embedded within clinical care across all secondary and tertiary hospitals in the region. This permitted us to include all consecutive patients who underwent testing for symptoms that were considered to be suggestive of COVID-19 by their usual care clinician minimizing selection bias and ensuring our findings are representative of all hospitalised patients across the region. The diagnosis was adjudicated by a multidisciplinary panel of clinicians from a range of specialities involved in the care of patients with COVID-19, including infectious disease, emergency medicine, general medicine and geriatric medicine, and therefore our findings are relevant to clinical practice across secondary and tertiary care settings.

To our knowledge this is the first evaluation of the diagnostic performance of the combined nasal and throat swab for a clinical diagnosis of COVID-19. The evaluation of the combined nasal and throat swab is particularly important since this is the most widely used diagnostic modality to identify or exclude SARS-CoV-2 infection. Although the RT-PCR test for SARS-CoV-2 has excellent in vitro analytical performance under carefully controlled laboratory conditions [9], diagnostic performance in clinical practice can vary due to multiple other factors, such as the site and quality of sampling, stage of disease, and viral multiplication or clearance $[3$, $6,13]$. Previous studies have reported diagnostic sensitivities ranging from 50 to $100 \%$ for the combined nasal and throat swab with a meta-analysis reporting a pooled meta-estimate of $89 \%$ (95\% CI 81 to $94 \%$ ) [4, 6, 14-17]. However, these studies have been performed in relatively small, selected patient cohorts, which limit the generalisability of study findings across the breadth of patients presenting to hospitals with suspected COVID-19. Furthermore, the reference standard used in these studies was either radiological findings on chest computed tomography, or the results of subsequent RT-PCR tests, which inevitably leads to an increase in the estimated diagnostic sensitivity. Indeed, when our reference standard was restricted to confirmed COVID-19 on serial testing, diagnostic sensitivity increased from 51.4 to $78.9 \%$.

In subgroup analyses, the diagnostic performance was similar in older patients, those with diabetes, known respiratory or cardiovascular disease. These findings are reassuring since these patient subgroups have been identified as those at the highest risk of death from COVID19 [18-20]. However, we observed that diagnostic sensitivity was lower in patients from the most deprived areas. This may reflect differences in the clinicians approach to testing by deprivation, rather than a consequence of deprivation itself, but this requires further evaluation. Furthermore, we observed that negative predictive value was lower in those who presented late in the course of their illness, and those with symptoms of fever and lower respiratory tract symptoms. This is consistent with virological assessments of patients hospitalised with COVID-19 which showed that viral RNA shedding from the upper respiratory tract is typically highest at the onset of symptoms, but subsequently declines as the disease progresses $[13,21]$. The lower negative predictive value in those with typical clinical symptoms is likely to reflect a higher pre-test probability for COVID-19 in these patients.

Our findings have potentially important implications for the use and interpretation of this test in clinical practice. Whilst the diagnosis of COVID-19 is still largely reliant on RT-PCR on material collected on nose and throat swabs, clinicians need to be aware of the strengths and limitations of testing when making decisions on the placement of patients within hospital settings and discharge planning. In our study, the most conservative estimate of diagnostic sensitivity was $51 \%$, where the index test was negative in 1 in 2 patients with the primary outcome of probable or confirmed COVID-19. Our most optimistic estimate of diagnostic sensitivity was $79 \%$, where the index test was negative in 1 in 5 patients with the secondary outcome of confirmed COVID-19. Whilst, the former may underestimate performance, the latter is certainly an overestimate due to circular reasoning, whereby the test under evaluation is an essential component of the reference standard.

In this consecutive series of hospitalised patients where testing was performed for symptoms at the discretion of the usual care clinician, our multi-disciplinary panel diagnosed probable COVID-19 in $12.6 \%$ of patients. The panel included representation from a broad range of medical specialities involved in the assessment of these patients, and therefore their judgment is likely to be representative of clinical practice. Our approach aims to provide insights into the performance of the test as it is applied in clinical practice. Interestingly patients with probable COVID-19 or confirmed COVID-19 and a negative index test were more likely to be retested 
within the next $24-48 \mathrm{~h}$. This likely reflects the usual care clinician's uncertainty when interpreting a negative test. Indeed for both the primary and secondary outcome, diagnostic performance improved significantly with up to four serial tests, and this observation could inform our approach to serial testing in practice with implications for patient flow and management of hospitalised patients. Our findings also have implications when defining the reference standard for studies evaluating the performance of point of care [22] and laboratory antibody tests [23] to determine those with and without prior infection. Future research should evaluate performance in patients undergoing testing in the community, and determine whether performance can be improved by incorporating a measure of sampling efficacy using epithelial cell counts [24], or whether the diagnostic yield is higher in other sample types, such as saliva or sputum.

We acknowledge our study has several limitations. We did not mandate the number of serial tests as all diagnostic testing was performed at the discretion of the treating clinician. Therefore those with a negative index test undergoing serial testing are likely to have had a higher pre-test probability than those undergoing a single test. Conversely, patients with a positive index test may have received testing due to uncertainty in the clinical diagnosis. Furthermore, during the conduct of this study, our institutional protocol recommended repeat testing when patients are transferred to different clinical areas; for example from a high dependency unit to a general ward. Nevertheless, it is reassuring that a similar increase in diagnostic performance with serial testing was observed in a sensitivity analysis restricted to those patients who had a complete series of at least four tests. Further studies with systematic sampling in consecutive patients are required to validate this observation. In the absence of an independent gold standard test for the diagnosis of COVID-19, our diagnostic evaluation was based on clinical review of all tests ordered by the usual care clinician. As a comprehensive panel of respiratory pathogens was not requested in all patients, it is likely we have misclassified some patients as COVID-19 who had other viral or bacterial infections. We may have overestimated the diagnosis of COVID-19 given the study was performed during the peak of a pandemic. However, we reviewed all available clinical investigations including all laboratory and imaging findings, and only defined patients with suspected COVID-19 where there was no alternative diagnosis. Furthermore, the clinical features of those with probable or confirmed COVID-19 were identical, suggesting no systematic bias was introduced during the adjudication.

\section{Conclusions}

In conclusion, a single combined nasal and throat swab with RT-PCR for SARS-CoV-2 has excellent specificity, but limited diagnostic sensitivity for the clinical diagnosis of COVID-19. Diagnostic performance is significantly improved by repeated testing.

\begin{abstract}
Abbreviations
ACE: Angiotensin converting enzyme; ALT: Alanine transaminase: ARB: Angiotensin receptor blockers; Cl: Confidence interval; COPD: Chronic obstructive pulmonary disease; COVID-19: Coronavirus disease 2019; IQR: Interquartile range; NPV: Negative predictive value; NHS: National Health Service; PCR: Polymerase chain reaction; PPV: Positive predictive value; RNA: Ribonucleic acid; RT-PCR: Reverse transcriptase-polymerase chain reaction; SARS-CoV-2: Severe acute respiratory syndrome coronavirus-2; SIMD: Scottish Index of Multiple Deprivation
\end{abstract}

\section{Supplementary Information}

The online version contains supplementary material available at https://doi. org/10.1186/s12879-021-05976-1.

Additional file 1: eTable 1. Virology, laboratory tests at presentation with suspected COVID-19. eTable 2. Baseline characteristics of patients stratified according to whether the diagnosis of COVID-19 was confirmed or probable. eTable 3. Virology, laboratory tests at presentation stratified according to whether the diagnosis of COVID-19 was confirmed or probable. eTable 4. Use of serial testing in patients with suspected COVID-19. eTable 5. Diagnostic performance of the index and serial combined nasal and throat swab for the secondary outcome of a diagnosis of confirmed COVID-19 on serial testing. eFigure 1. Stack plot of the number of RT-PCR tests performed stratified according to whether the test was positive (red) or negative (blue). eFigure 2. Heat map of RT-PCR testing in patients with confirmed COVID-19 stratified according to whether the index test was negative (a) or positive (b). eFigure 3. Sensitivity of serial testing using the combined nasal and throat swab for the primary (confirmed and probable COVID-19) and secondary (confirmed COVID-19) outcome in patients who were tested at least four times. eFigure 4. Negative predictive value of serial testing using the combined nasal and throat swab for the primary (confirmed and probable COVID-19) and secondary (confirmed COVID-19) outcome in patients who were tested at least four times. eFigure 5. Forest plot of the (a) sensitivity and (b) negative predictive value of the index combined nasal and throat swab for a diagnosis of confirmed COVID-19 stratified by subgroups.

\section{Acknowledgements}

Not applicable.

DataLoch COVID-19 Collaborators

Clinical Lead: Atul Anand.

Programme Lead: Kathy Harrison.

DataLoch Technical and Management Group: Catherine Stables, Ally Hume, David Homan, Catriona Waugh, Jilly McKay, Chris Duncan, Ronnie Harkess.

DataLoch Leadership Group: Kathy Harrison, Michael Gray, Colan Mahaffey, Pamela Linksted, Atul Anand, Anoop SV Shah, Rob Baxter, Peter Cairns, Nicola Rigglesford, Martin Egan, Nicholas L Mills.

Infectious Diseases Unit: Daniella A Ross, Claire L Mackintosh, Oliver Koch, Kate Templeton, Meghan R Perry.

COVID-19 Clinical Data Review: Daniella A Ross, Anda Bularga, Hannah MM Preston, Thomas J McCormick, Arjuna A Sivakumaran, Kathryn AW Knight, Rosie Callender, Anna K Jamieson, Jonathan Wubetu, John P Kelly, Zaina Sharif, Ha Bao Trung Le, Jason Yang, Arun Parajuli, Ed Whittaker, Oscar CN Maltby, Sarah H Goodwin, Louisa R Cary, Emma K Watson, Thomas H Clouston, Julia Guerrero Enriquez, XinYi Ng.

COVID-19 Adjudication Panel: Kuan Ken Lee, Daniella A Ross, Anda Bularga, Andrew R Chapman, Yvonne K McFarlane, Kate H Regan, Richard P Biggers, John P Kelly, Kathryn AW Knight, Hannah MM Preston, Thomas J McCormick, Anoop SV Shah, Atul Anand, Meghan R Perry, Nicholas L Mills.

NHS Lothian eHealth and Lothian Analytical Services: Alistair Stewart, Alastair Thomson, Chris Duncan, Daniella Ene, Hazel Neilson, Juergen Caris, Maria McMenemy, Nazir Lone, Nicola Rigglesford, Paul Schofield, Sophie McCall, Stephen Young, Tracey McKinley, Tracey Rapson. 


\section{Authors' contributions}

KKL, AA, ASVS and NLM conceived the study and its design. KKL, DR, AB, $A R C, A S V S, A A, M P$, and NLM acquired the data. KKL and DD performed the analysis. KKL, DD, DR, AB, CM, OK, IJ, SJ, KT, ARC, ASVS, AA, MP, and NLM interpreted the data. KKL and NLM drafted the manuscript. All authors revised the manuscript critically for important intellectual content and provided their final approval of the version to be published. All authors are accountable for the work.

\section{Funding}

This study was funded by a British Heart Foundation (BHF) Research Excellence Award (RE/18/6134217). DataLoch ${ }^{\text {TM }}$ is funded by the University of Edinburgh, and the UK and Scottish Governments as part of the Data Driven Innovation in Health \& Social Care programme. AA is supported by a Clinical Lectureship from the Chief Scientist Office (PCL/18/05). KL and NLM are supported by a Clinical Research Training Fellowship (FS/18/25/33454) and the Butler Senior Clinical Research Fellowship (FS/16/14/32023) from the British Heart Foundation, respectively. DD is supported by the Medical Research Council (MR/N013166/1). AB is supported by Clinical Research Training Fellowships from the Medical Research Council (MR/N007254/1). ARC is supported by a Starter Grant for Clinical Lecturers from the Academy of Medical Sciences (SGL021\1075).

The funders played no role in the study design, in the collection, analysis, and interpretation of the data, in the writing of the report, or in the decision to submit the paper for publication. The authors had full access to all the data in the study and had final responsibility for the decision to submit for publication.

\section{Availability of data and materials}

The datasets used and/or analysed during the current study are available from the corresponding author on reasonable request.

\section{Declarations}

\section{Ethics approval and consent to participate}

The study was performed with approval of the local Research Ethics Committee and delegated Caldicott Guardian for the National Health Service (NHS) Lothian Health Board, in accordance with the Declaration of Helsinki. All data were collected from the patient record and national registries, deidentified and linked in a data repository (DataLoch, Edinburgh, United Kingdom) within a secure safe haven. To ensure that every eligible patient was included and avoid selection bias, individual patient consent was waived with approval from our research ethics committee and delegated Caldicott Guardian. Only summary data was extracted to minimise the risk of disclosure.

\section{Consent for publication}

Not applicable.

\section{Competing interests}

All authors have no interests to declare.

\section{Author details}

${ }^{1}$ BHF Centre for Cardiovascular Science, University of Edinburgh, Edinburgh EH16 4SA, UK. 'Usher Institute, University of Edinburgh, Edinburgh, UK. ${ }^{3}$ Regional Infectious Disease Unit, Western General Hospital, Edinburgh, UK. ${ }^{4}$ Department of Clinical Virology, Royal Infirmary of Edinburgh, Edinburgh, UK. ${ }^{5}$ Department of Clinical Biochemistry, Royal Infirmary of Edinburgh, Edinburgh, UK. ${ }^{6}$ Department of Non-communicable Disease Epidemiology, London School of Hygiene and Tropical Medicine, London, UK. ${ }^{7}$ Department of Cardiology, Imperial College Healthcare NHS Trust, London, UK.

\section{Received: 29 October 2020 Accepted: 9 March 2021}

\section{Published online: 06 April 2021}

\section{References}

1. Huang C, Wang Y, Li X, Ren L, Zhao J, Hu Y, Zhang L, Fan G, Xu J, Gu X, Cheng Z, Yu T, Xia J, Wei Y, Wu W, Xie X, Yin W, Li H, Liu M, Xiao Y, Gao H, Guo L, Xie J, Wang G, Jiang R, Gao Z, Jin Q, Wang J, Cao B. Clinical features of patients infected with 2019 novel coronavirus in Wuhan, China. Lancet. 2020;395(10223):497-506. https://doi.org/10.1016/S0140-6736(20)30183-5.
2. Gates B. Responding to Covid-19 - a once-in-a-century pandemic? N Engl J Med. 2020;382(18):1677-9. https://doi.org/10.1056/NEJMp2003762.

3. Sethuraman $N$, Jeremiah SS, Ryo A. Interpreting diagnostic tests for SARSCoV-2. JAMA. 2020;323(22):2249-51. https://doi.org/10.1001/jama.2020.8259.

4. Fang $Y$, Zhang $H$, Xie J, Lin M, Ying L, Pang P, Ji W. Sensitivity of chest CT for COVID-19: comparison to RT-PCR. Radiology. 2020;296(2):E115-E117.

5. Guan WJ, Ni ZY, Hu Y, Liang WH, Ou CQ, He JX, Liu L, Shan H, Lei CL, Hui DSC, du B, Li L, Zeng G, Yuen KY, Chen RC, Tang CL, Wang T, Chen PY, Xiang J, Li SY, Wang JL, Liang ZJ, Peng YX, Wei L, Liu Y, Hu YH, Peng P, Wang JM, Liu JY, Chen Z, Li G, Zheng ZJ, Qiu SQ, Luo J, Ye CJ, Zhu SY, Zhong NS, China Medical Treatment Expert Group for Covid-19. Clinical characteristics of coronavirus disease 2019 in China. N Engl J Med. 2020; 382(18):1708-20. https://doi.org/10.1056/NEJMoa2002032.

6. Wang W, Xu Y, Gao R, Lu R, Han K, Wu G, Tan W. Detection of SARS-CoV-2 in different types of clinical specimens. JAMA. 2020;323(18):1843-4.

7. Pan X, Chen D, Xia Y, Wu X, Li T, Ou X, Zhou L, Liu J. Asymptomatic cases in a family cluster with SARS-CoV-2 infection. Lancet Infect Dis. 2020;20(4):4101. https://doi.org/10.1016/S1473-3099(20)30114-6.

8. Bai Y, Yao L, Wei T, Tian F, Jin DY, Chen L, Wang M. Presumed asymptomatic carrier transmission of COVID-19. JAMA. 2020;323(14):1406-7. https://doi.org/10.1001/jama.2020.2565.

9. Corman VM, Landt O, Kaiser M, Molenkamp R, Meijer A, Chu DK, Bleicker T, Brunink S, Schneider J, Schmidt ML, et al. Detection of 2019 novel coronavirus (2019-nCoV) by real-time RT-PCR. Euro Surveill. 2020;25(3).

10. Williams T, Wastnedge E, McAllister G, Bhatia R, Cuschieri K, Kefala K, Hamilton F, Johannessen I, Laurenson I, Shepherd J, et al. Sensitivity of RTPCR testing of upper respiratory tract samples for SARS-CoV-2 in hospitalised patients: a retrospective cohort study [version 1; peer review: 1 approved]. Wellcome Open Res. 2020;5(254).

11. The Scottish Index of Multiple Deprivation. Scottish Government. (https:// www2.gov.scot/Topics/Statistics/SIMD) [Accessed 8 July 2020].

12. Public health surveillance for COVID-19. Interim guidance. World Health Organisation. 2020.

13. Wolfel R, Corman VM, Guggemos W, Seilmaier M, Zange S, Muller MA, Niemeyer D, Jones TC, Vollmar P, Rothe C, et al. Virological assessment of hospitalized patients with COVID-2019. Nature. 2020;581(7809):465-9. https://doi.org/10.1038/s41586-020-2196-x.

14. Kim H, Hong H, Yoon SH. Diagnostic Performance of $C T$ and Reverse Transcriptase-Polymerase Chain Reaction for Coronavirus Disease 2019: A Meta-Analysis. Radiology. 2020:201343.

15. Wu C, Chen X, Cai Y, Xia J, Zhou X, Xu S, Huang H, Zhang L, Zhou X, Du C, et al. Risk factors associated with acute respiratory distress syndrome and death in patients with coronavirus disease 2019 pneumonia in Wuhan, China. JAMA Intern Med. 2020;180(7):934-43.

16. Li Y, Yao L, Li J, Chen L, Song Y, Cai Z, Yang C. Stability issues of RT-PCR testing of SARS-CoV-2 for hospitalized patients clinically diagnosed with COVID-19. J Med Virol. 2020;92(7):903-8. https://doi.org/10.1002/jmv.25786.

17. Ai T, Yang Z, Hou H, Zhan C, Chen C, Lv W, Tao Q, Sun Z, Xia L. Correlation of chest CT and RT-PCR testing in coronavirus disease 2019 (COVID-19) in China: a report of 1014 cases. Radiology. 2020;200642.

18. Docherty AB, Harrison EM, Green CA, Hardwick HE, Pius R, Norman L, Holden KA, Read JM, Dondelinger F, Carson G, et al. Features of 20133 UK patients in hospital with covid-19 using the ISARIC WHO clinical characterisation protocol: prospective observational cohort study. BMJ. 2020:369.

19. Du RH, Liang LR, Yang CQ, Wang W, Cao TZ, Li M, Guo GY, Du J, Zheng CL, Zhu Q, et al. Predictors of mortality for patients with COVID-19 pneumonia caused by SARS-CoV-2: a prospective cohort study. Eur Respir J. 2020;55(5).

20. Williamson EJ, Walker AJ, Bhaskaran K, Bacon S, Bates C, Morton CE, Curtis HJ, Mehrkar A, Evans D, Inglesby P, Cockburn J, McDonald HI, MacKenna B, Tomlinson L, Douglas IJ, Rentsch CT, Mathur R, Wong AYS, Grieve R, Harrison D, Forbes H, Schultze A, Croker R, Parry J, Hester F, Harper S, Perera R, Evans SJW, Smeeth L, Goldacre B. OpenSAFELY: factors associated with COVID-19 death in 17 million patients. Nature. 2020;584(7821):430-6. https://doi.org/10.1038/s41586-020-2521-4.

21. Zheng S, Fan J, Yu F, Feng B, Lou B, Zou Q, Xie G, Lin S, Wang R, Yang X, et al. Viral load dynamics and disease severity in patients infected with SARS-CoV-2 in Zhejiang province, China, January-march 2020: retrospective cohort study. BMJ. 2020;369.

22. Li Z, Yi Y, Luo X, Xiong N, Liu Y, Li S, Sun R, Wang Y, Hu B, Chen W, et al. Development and clinical application of a rapid IgM-lgG combined 
antibody test for SARS-CoV-2 infection diagnosis. Med Virol. 2020;92(9): $1518-24$.

23. Long QX, Liu BZ, Deng HJ, Wu GC, Deng K, Chen YK, Liao P, Qiu JF, Lin Y, Cai XF, Wang DQ, Hu Y, Ren JH, Tang N, Xu YY, Yu LH, Mo Z, Gong F, Zhang XL, Tian WG, Hu L, Zhang XX, Xiang JL, du HX, Liu HW, Lang CH, Luo XH, Wu SB, Cui XP, Zhou Z, Zhu MM, Wang J, Xue CJ, Li XF, Wang L, Li ZJ, Wang K, Niu CC, Yang QJ, Tang XJ, Zhang Y, Liu XM, Li JJ, Zhang DC, Zhang F, Liu P, Yuan J, Li Q, Hu JL, Chen J, Huang AL. Antibody responses to SARSCoV-2 in patients with COVID-19. Nat Med. 2020;26(6):845-8. https://doi. org/10.1038/s41591-020-0897-1.

24. Bonnin P, Miszczak F, Kin N, Resa C, Dina J, Gouarin S, Viron F, Morello R, Vabret A. Study and interest of cellular load in respiratory samples for the optimization of molecular virological diagnosis in clinical practice. BMC Infect Dis. 2016;16(1):384. https://doi.org/10.1186/s12879-016-1730-9.

\section{Publisher's Note}

Springer Nature remains neutral with regard to jurisdictional claims in published maps and institutional affiliations.

Ready to submit your research? Choose BMC and benefit from:

- fast, convenient online submission

- thorough peer review by experienced researchers in your field

- rapid publication on acceptance

- support for research data, including large and complex data types

- gold Open Access which fosters wider collaboration and increased citations

- maximum visibility for your research: over $100 \mathrm{M}$ website views per year

At $\mathrm{BMC}$, research is always in progress.

Learn more biomedcentral.com/submissions 